\title{
Impact of demographics on human gut microbial diversity in a US Midwest population
}

Jun Chen, Euijung Ryu, Matthew Hathcock, Karla Ballman, Nicholas Chia, Janet E Olson, Heidi Nelson

The clinical utility of microbiome biomarkers depends on the reliable and reproducible nature of comparative results. Underappreciation of the variation associated with common demographic, health, and behavioral factors may confound associations of interest and generate false positives. Here, we present the Midwestern Reference Panel (MWRP), a resource for comparative gut microbiome studies conducted in the Midwestern United States. We analyzed the relationships between demographic and health behavior-related factors and the microbiota in this cohort, and estimated their effect sizes. Most variables investigated were associated with the gut microbiota. Specifically, body mass index (BMI), race, sex, and alcohol use were significantly associated with microbial $\beta$-diversity $(P<$ 0.05 , unweighted UniFrac). BMI, race and alcohol use were also significantly associated with microbial $\alpha$-diversity ( $P<0.05$, species richness). Tobacco use showed a trend toward association with the microbiota ( $P<0.1$, unweighted UniFrac). The effect sizes of the associations, as quantified by adjusted $\mathrm{R}^{2}$ values based on unweighted UniFrac distances, were small ( $<1 \%$ for all variables), indicating that these factors explain only a small percentage of overall microbiota variability. Nevertheless, the significant associations between these variables and the gut microbiota suggest that they could still be potential confounders in comparative studies and that controlling for these variables in study design, which is the main objective of the MWRP, is important for increasing reproducibility in comparative microbiome studies. 


\section{Impact of demographics on human gut microbial diversity in a

3 Jun Chen ${ }^{1,2}$, Euijung Ryu ${ }^{2}$, Matthew Hathcock ${ }^{2}$, Karla Ballman ${ }^{2,3}$, Nicholas Chia ${ }^{1,2,4,5}$, Janet

4 Olson $^{2}$, Heidi Nelson ${ }^{1,4}$

$5{ }^{1}$ Microbiome Program, Center for Individualized Medicine, Mayo Clinic, Rochester MN 55905, 6 U.S.A.

$7 \quad 2$ Department of Health Sciences Research, Mayo Clinic, Rochester MN 55905, U.S.A.

$8{ }^{3}$ Division of Biostatistics and Epidemiology, Weill Medical College of Cornell University, New 9 York NY 10065, U.S.A.

$10{ }^{4}$ Department of Surgery, Mayo Clinic, Rochester MN 55905, U.S.A.

115 Department of Physiology and Biomedical Engineering, Mayo Clinic, Rochester MN 55905, 12 U.S.A

14 Corresponding Author:

15 Heidi Nelson

162001 st St SW

17 Rochester, MN 55902, U.S.A.

18 Email: nelsonh@mayo.edu

Running title: Human demographics and gut microbial diversity 
Abstract

The clinical utility of microbiome biomarkers depends on the reliable and reproducible nature of comparative results. Underappreciation of the variation associated with common demographic, health, and behavioral factors may confound associations of interest and generate false positives. Here, we present the Midwestern Reference Panel (MWRP), a resource for comparative gut microbiome studies conducted in the Midwestern United States. We analyzed the relationships between demographic and health behavior-related factors and the microbiota in this cohort, and estimated their effect sizes. Most variables investigated were associated with the gut microbiota. Specifically, body mass index (BMI), race, sex, and alcohol use were significantly associated with microbial $\beta$-diversity ( $\mathrm{P}<0.05$, unweighted UniFrac). BMI, race and alcohol use were also significantly associated with microbial $\alpha$-diversity $(\mathrm{P}<0.05$, species richness). Tobacco use showed a trend toward association with the microbiota $(\mathrm{P}<0.1$, unweighted UniFrac $)$. The effect sizes of the associations, as quantified by adjusted $\mathrm{R}^{2}$ values based on unweighted UniFrac distances, were small ( $<1 \%$ for all variables), indicating that these factors explain only a small percentage of overall microbiota variability. Nevertheless, the significant associations between these variables and the gut microbiota suggest that they could still be potential confounders in comparative studies and that controlling for these variables in study design, which is the main objective of the MWRP, is important for increasing reproducibility in comparative microbiome studies.

\section{Introduction}

Humans are populated by a vast number of microbes. It is estimated that bacterial cells associated with the human body outnumber human cells by a factor of 10 (Cho \& Blaser, 2012). These microbes and their genetic content constitute the human microbiome (Cho \& Blaser, 2012). The gut microbiome alone accounts for more than three million genes, more than 100 times the number of human genes (Qin et al., 2010). The microbiome has both digestive and metabolic functions (Cani \& Delzenne, 2009) and plays an important role in the development of the host immune system (Round \& Mazmanian, 2009). Extensive evidence shows that a core microbiome is responsible for maintaining a healthy state; any significant deviation may affect 
51 an individual's risk of disease (Sartor, 2004; Ley et al., 2006; Huse et al., 2012; Human 52 Microbiome Project Consortium, 2012; Zhang et al., 2015)

Recognition of the microbiome's importance to human health and disease has inspired a surge of studies evaluating changes in the gut microbiota in various conditions, including cancer, inflammatory diseases, and obesity (Turnbaugh et al., 2006; Qin et al., 2012; Scher et al., 2013; David, 2013; Schubert et al., 2014; Abreu \& Peek, 2014). To adequately evaluate the changes associated with these conditions, a truly representative control group must be used. However, the definition of an ideal control group is complicated by the complexity of the microbiota and its extensive inter- and intra-individual variation. Historically, it has been presumed that young adults who have no identified and/or treated medical conditions are ideally suited for establishing normative laboratory values. This approach reduces confounding influences and provides an opportunity to consider what the structure of a "healthy" adult microbial community might look like. Therefore, attempts have been made to generate and characterize cohorts of "healthy" individuals, with the intention of developing standard reference microbiomes for use in future studies. The Human Microbiome Project (HMP) and the MetaHIT represent two such large-scale endeavors (Qin et al., 2010; Gevers et al., 2012; Foxman \& Rosenthal, 2013). However, "healthy" individuals may not accurately represent an epidemiologic population with diverse health conditions. Moreover, the distribution of demographic variables in such a group may be different from that of the epidemiologic population, creating the potential for confounding in comparative studies.

In epidemiologic studies of US Midwestern populations at our institute, the Mayo Clinic, normative values are generated, validated, and used to help guide diagnostic and therapeutic purposes. These normative values typically apply to broad populations of people with diverse health conditions, such that a lab value outside the normative range suggests a specific condition or a set of conditions. Here, we apply this approach to the microbiome, offering a broad Midwestern Reference Panel (MWRP) for use in disease association studies of Midwestern populations.

The objectives of the current study were twofold. First, we aimed to create a representative epidemiologic sample from the Midwestern United States, excluding subjects with conditions that would directly impact the gut microbiota, such as gastrointestinal (GI) disorders. 
81 Second, we aimed to study the effect sizes of demographic and health behavior-related variables, including sex, age, race, BMI, alcohol use, and smoking habits, on the gut microbiota. Thus, in addition to introducing a truly representative cohort for the Midwestern United States population, this study reveals which demographic and health behavior-related variables have the greatest effect on the gut microbiome and, therefore, should be given special attention in study design and the interpretation of results.

\section{Materials and Methods}

Biobank/Consent. Subjects were selected from the Mayo Clinic Biobank, an institutional resource composed of biological specimens accompanied by clinical data obtained from patient medical records and patient-provided risk factor data (Olson et al., 2013). Written informed consent was provided by all individuals enrolled. The study was approved by the Institutional Review Board of the Mayo Clinic (\#13-003694).

Subject Selection/Questionnaire. As described below, representative fecal samples were chosen according to sex, age, race, body mass index (BMI), alcohol use, and tobacco use. A survey accompanied the invitation to participate in the study and inquired about bowel symptoms, such as diarrhea, constipation, blood in the stool, and nausea or vomiting; current medication use; current supplement use; antibiotic use in the past 2 weeks; current smoking status; current alcohol intake; current weight; and cancer history.

To define a sample truly representative of the Midwestern United States (Minnesota, Iowa, North Dakota, South Dakota, and Wisconsin), we estimated the distributions of selected behavioral risk factors among subjects included in the Center for Disease Control's 2011 Behavioral Risk Factor Surveillance Survey (BRFSS; http://www.cdc.gov/brfss/annual_data/annual_2011.htm). Mayo Clinic Biobank participants were then selected based upon BRFSS distributions of age, sex, race, BMI, smoking status, and alcohol intake. Two rounds of subject selection were employed for this study. The first round took place in the summer of 2013. During this round, 380 subjects (ages 20-49 years) were selected. In the summer of 2014, a second group of 267 patients (ages 50-79 years) was selected. Stool samples were collected from $25 \%$ of the samples who consented in the first round and $31 \%$ of the subjects in the second round. 
The final, combined sample was chosen on the basis of age $(20-29,30-39,40-49,50-$ 59, 60-69, and 70-79 year groups), sex (10 males and 10 females in each age group), race (white or non-white), BMI ( $<30 \mathrm{~kg} / \mathrm{m}^{2}$ or $\left.\geq 30 \mathrm{~kg} / \mathrm{m}^{2}\right)$, smoking status (current or former/never), and alcohol use (yes or no). The proportions of all inclusion criteria were chosen to reflect the underlying population in the Upper Midwestern states, as estimated by data from the BRFSS. Exclusion criteria, assessed based on survey information completed at the time of enrollment into the study, included use of medications, non-GI cancers, use of any antibiotic within the past 2 weeks, or any known GI disease or symptoms including, but not limited to, inflammatory bowel disease, irritable bowel syndrome, esophageal cancer, intestinal cancer, constipation, diarrhea, Clostridium difficile infection, and celiac disease/sprue.

Collection method. The collection process was reviewed in detail with study participants, and a stool collection kit was provided to each subject. Stool samples were collected by the subjects and returned to Mayo Clinic Rochester within 24 hours of passing. Specimens were stored at $-80^{\circ} \mathrm{C}$ until DNA extraction.

Sample preparation and sequencing. Fecal DNA was extracted using the PowerSoil kit

125 (MoBio, Carlsbad, CA) according to the manufacturer's instructions. Genomic DNA was used 126 as a template for the polymerase chain reaction (PCR), with $0.3 \mu \mathrm{M} \mathrm{V3-V5} \mathrm{barcoded} \mathrm{primers}$

127 (Caporaso et al., 2012) targeting 357F and 926R of the bacterial 16S gene

128 (5'AATGATACGGCGACCACCGAGATCTACACTATGGTAATTGTCCTACGGGAGGCAG 129 CAG3' and

130 5'CAAGCAGAAGACGGCATACGAGATNNNNNNNNNNNNAGTCAGTCAGCCCCGTCA

131 ATTCMTTTRAGT3', respectively). PCR conditions were as follows: $95^{\circ} \mathrm{C} / 3 \mathrm{~min}$; 35 cycles of $13298^{\circ} \mathrm{C} / 30 \mathrm{~s}, 70^{\circ} \mathrm{C} / 15 \mathrm{~s}$, and $72^{\circ} \mathrm{C} / 15 \mathrm{~s}$; and finally $72^{\circ} \mathrm{C} / 5 \mathrm{~min}$ in a Bio-Rad $\mathrm{T} 100$ Thermocycler 133 (Hercules, CA) using Kapa Hotstart Hi-Fi DNA polymerase (Kapa Biosystems, Boston, MA).

134 PCR product sizes were verified using the Agilent TapeStation with reaction cleanup, and DNA 135 was purified using an epMotion automated system (Eppendorf, Hauppauge, NY) with the 136 Agencourt AMPure PCR Purification System (Beckman Coulter, Brea, CA). Final quantitation 137 was performed using a QuBit HS dsDNA kit and the QuBit 2.0 fluorimeter (Life Technologies, 138 Carlsbad, CA). Samples were pooled to equal concentration and sequenced on one lane of a 139 MiSeq at the Mayo Genomics Facility using the MiSeq Reagent Kit v2 $(2 \times 250$ reads, 500 
140 cycles; Illumina Inc., San Diego, CA). Pre-processed sequence files were then processed via the 141 IM-TORNADO bioinformatics pipeline with the default parameter settings to form operational taxonomic units (OTUs) (Jeraldo et al., 2014). IM-TORNADO uses paired-end reads to form OTUs, and was shown to be more sensitive than methods using single-end reads based on synthetic mock community studies.

Statistical analyses. Statistical analysis proceeded in two steps. First, overall associations between demographic and health behavior-related variables and the microbiota were investigated. Next, specific associations at the level of taxa were investigated.

To perform overall association tests, we summarized microbiota data using both $\alpha$ diversity and $\beta$-diversity. $\alpha$-diversity reflects species richness and evenness within bacterial populations. Two $\alpha$-diversity metrics, the observed OTU number and the Shannon index, were investigated. The observed OTU number reflects species richness, whereas the Shannon index measures both species richness and evenness. Before calculating the $\alpha$-diversity metrics, we first replaced the observed singleton OTU count with a more robust estimate to reduce the influence of sequencing errors (Chiu \& Chao, 2015). We then computed the $\alpha$-diversity estimates at the minimum sample coverage among all samples (0.997) to standardize by sample completeness (“estimateD” in the R package iNEXT) (Chao et al., 2014). $\beta$-diversity reflects the shared diversity between bacterial populations in terms of ecological distance; different distance metrics provide distinctive views of community structure. Two $\beta$-diversity measures, unweighted and weighted UniFrac distances, were calculated using the OTU table and a phylogenetic tree (“GUniFrac” function in the R package GUniFrac) (Lozupone \& Knight, 2005; Chen et al., 2012). The unweighted UniFrac reflects differences in community membership (i.e., the presence or absence of an OTU), whereas the weighted UniFrac captures this information and also differences in abundance. Rarefaction was performed on the OTU table before calculating UniFrac distances. model:

$$
\text { Microbiota } \sim \text { Batch }+\mathrm{X}+\mathrm{X}: \text { Batch }
$$


168

169

170

171

172

173

174

175

176

177

178

179

180

181

182

183

184

185

186

where microbiota is the outcome variable, summarized by the diversity measures described above, and $\mathrm{X}$ is the variable of interest. In the model, age and BMI were treated as continuous variables while the other variables as categorical. We included a batch variable to account for potential batch effects, since sequencing was performed in two batches, and a covariate-Batch (X:Batch) interaction term. The interaction term allowed different association strengths for the two batches. We assessed the overall effect (main + interaction effect), which is equivalent to testing the null hypothesis of no association in either batch. To assess the association with $\alpha$ diversity measures, we performed regular linear regression analysis with the likelihood ratio test of regression coefficients, as the outcome was approximately normal. To assess the association with $\beta$-diversity measures, we used the PERMANOVA procedure ("adonis" function in the $\mathrm{R}$ package vegan), which is a multivariate analysis of variance based on distance matrices and permutation (McArdle \& Anderson, 2001). Using the PERMANOVA procedure, statistical significance was assessed by comparing the observed association between microbiota composition and a variable of interest with associations for 1,000 random permutations.

Ordination plots were generated using principal coordinate analysis ( $\mathrm{PCoA})$ on unweighted UniFrac-based distances as implemented in R ("cmdscale" function in the standard R package).

A distance-based coefficient of determination, $\mathrm{R}^{2}$ (i.e., the percentage of overall microbiota variability explained by a variable), was used to quantify the effect size of the overall association (McArdle \& Anderson, 2001). Distance-based $\mathrm{R}^{2}$ is defined as

$$
\mathrm{R}^{2}=\frac{\operatorname{tr}(\mathrm{HGH})}{\operatorname{tr}(\mathrm{G})},
$$

where $\operatorname{tr}($.$) is the trace of a matrix, \mathrm{H}$ is the projection matrix into the column space spanned by the corresponding variable, and $\mathrm{G}$ is the Gower's centered matrix, which is defined as

$$
G=\left(I-\frac{11^{T}}{n}\right) A\left(I-\frac{11^{T}}{n}\right),
$$

where $\mathrm{I}$ is an identity matrix, 1 is a vector of $1 \mathrm{~s}$, and $\mathrm{A}=\left(\mathrm{a}_{\mathrm{ij}}\right)_{\mathrm{nxn}}=\left(-\mathrm{d}_{\mathrm{ij}}^{2} / 2\right)_{\mathrm{nxn}}$ is a matrix constructed using the pairwise distances $\mathrm{d}_{\mathrm{ij}}$. In the formula, the total variability of the microbiota was summarized using a distance metric. To quantify the overall effect size well, a good distance metric should capture the association signals. In this study, we used the unweighted UniFrac distance, as most of the associations investigated were significant only for this metric. To 
196 account for potential overestimation due to a small sample size, we calculated an adjusted $\mathrm{R}^{2}$,

197 which is defined as

198

$$
\mathrm{R}_{\text {adj }}^{2}=1-\frac{\left(1-\mathrm{R}^{2}\right)(\mathrm{n}-1)}{\mathrm{n}-\mathrm{p}-1},
$$

where $\mathrm{n}$ is the sample size and $\mathrm{p}$ is the degree of freedom of the covariate.

Next, we assessed associations between demographic and health behavior-related factors

201

202

203

204

205

206

207

208

209

210

211

212

213

214

215

216

217

218

219

220

221

222

\section{Results}

\section{Gut microbiota profile of the Midwestern United States}

As revealed by a comparison with BRFSS data, the distribution of important demographic variables within the MWRP cohort, which consists of 118 subjects drawn from the Mayo Clinic Biobank, generally reflects the demographic characteristics of the Midwestern United States, although alcohol users are slightly over-represented in subjects older than 50 years of age (Table $1)$.

Stool samples from this cohort were collected and deep sequenced. 16S rDNA-targeted sequencing yielded 110,997 reads/sample on average (range: 33,579-385,720). Clustering of these $16 \mathrm{~S}$ sequence tags produced 1,745 non-singleton OTUs at a 97\% similarity level. The distribution of OTU abundance and prevalence was typical, dominated by rare and lowabundance OTUs (Fig. 1A, B). An average of 402 OTUs was detected in each subject (range: 186-810). The median OTU abundance was 163 read counts per OTU (range: 2-1,456,439), and 
224 the median OTU prevalence was $11 \%$ (range: $0.8 \%-100 \%)$. Only 61 OTUs (3.5\% of total OTUs)

225 occurred in more than $95 \%$ of samples, indicating a relatively small core set of OTUs. The

226 detected OTUs were classified into 11 phyla, 80 families, and 171 genera using the Ribosomal

227 Database Project (RDP) classifier. At the phylum level, Firmicutes accounted for $48.3 \%$ of total

228 reads and Bacteroides for $48.1 \%$ of total reads. At the family level, the most abundant families

229 were Bacteroidaceae (33.76\%), Ruminococcaceae (22.94\%), and Lachnospiraceae (15.13\%). At

230 the genus level, the dominant genera were Bacteroides (33.76\%), Ruminococcus (9.83\%), and

231 Faecalibacterium (9.13\%). Consistent with previous studies, we observed large intersubject

232 variability in taxa abundance (Fig. 1C, D) (Human Microbiome Project Consortium, 2012;

233 Flores et al., 2014). Table 2 lists the "core" taxa (prevalence greater than 95\%) identified in this

234 study; we identified 4 core phyla, 18 core families, and 22 core genera. The overall pattern in this

235 cohort was similar to that in the HMP, although specific values were very different (Huse et al.,

236 2012; Human Microbiome Project Consortium, 2012). This was probably due to biological

237 variability, including differences in genetics, demographics, and health behavior, as well as

238 technical variability, including differences in sample collection, preparation, sequencing, and

239 bioinformatics processing.

\section{Demographic and health behavior-related variables shaping the gut microbiota}

241 Because of the wide demographic and health behavior-related variation it captures, the MWRP data set provides a good opportunity to identify associations between these variables and the microbiota. We first performed overall association tests based on $\alpha$ - and $\beta$-diversity measures. $\alpha$ and $\beta$-diversity measures provide a holistic view of the microbiota; distinctive yet interrelated, these measures focus on different aspects of microbiota structure. In this study, we chose the following $\alpha$-diversity measures: the number of observed OTUs (after rarefaction) as a species richness measure, and the Shannon index as an overall diversity measure incorporating both species richness and abundance. In terms of $\beta$-diversity, which describes overall microbiota structure, we chose the phylogeny-based unweighted and weighted UniFrac distance metrics; the unweighted UniFrac focuses on community membership, whereas the weighted UniFrac reflects both membership and abundance (Chen et al., 2012). 

associated with decreased species richness $(\mathrm{P}=0.006$, Fig. $2 \mathrm{~A})$. BMI was also significantly associated with overall microbiota structure, as revealed by both unweighted and weighted UniFrac analysis $(\mathrm{P}=0.006$ and 0.030 , Table 4$)$. Race was associated with both species richness $(\mathrm{P}=0.013$, Fig. 2B) and overall microbiota structure $(\mathrm{P}=0.003$, unweighted UniFrac $)$, with white subjects exhibiting greater species richness. Sex and alcohol use were both associated with overall structure $(\mathrm{P}=0.049$ and 0.041 respectively, unweighted UniFrac, Fig 2C, D). In addition, alcohol use was associated with increased species richness $(\mathrm{P}=0.040$, Table 3$)$. Some evidence of association of tobacco use with overall microbiota structure was observed $(\mathrm{P}=0.091$, unweighted UniFrac). Age, by contrast, was not significantly associated with measures of $\alpha$ - or $\beta$-diversity. Statistical significance was primarily observed for unweighted measures, such as species richness and the unweighted UniFrac distance, indicating that these demographic and health behavior-related factors chiefly affect community membership or the rare biosphere of the microbiota (Chen et al., 2012).

Next, we quantified the effect sizes of these variables with adjusted, distance-based $\mathrm{R}^{2}$ values (see Methods). Using the unweighted UniFrac distance to summarize overall microbiota variability, the adjusted $\mathrm{R}^{2}$ values were $0.95 \%$ for race, $0.88 \%$ for BMI, $0.53 \%$ for alcohol use, $0.46 \%$ for sex, $0.33 \%$ for tobacco use, and $0.0 \%$ for age (Fig. 3), indicating huge intersubject variability relative to the variability associated with these factors.

\section{Microbial signature of demographic and health behavior-related factors}

We next set out to identify the microbial signature of the demographic and behavioral factors that were most significantly associated with overall microbiota structure (BMI, sex, race, tobacco use, and alcohol use). We used permutation-based univariate association tests to identify associated bacterial taxa at the phylum, family, and genus level. FDR control was used to correct for multiple testing.

Consistent with overall association tests, a number of bacterial taxa were associated with BMI at an FDR of 10\% (Fig. 4, Table 5). In particular, we observed an increase in Firmicutes and a decrease in Bacteroidetes in obese subjects, consistent with findings from some previous studies (Turnbaugh et al., 2009; Walters, Xu \& Knight, 2014). In addition, we detected an 
281 increase in the genus Eubacterium, consistent with two previous studies that reported

282 associations between Eubacterium rectale and obesity (Walters, Xu \& Knight, 2014).

283 Interestingly, we also found an increase in Fusobacteria, which have been shown to be associated 284 with colon cancer (Rubinstein et al., 2014).

285

286

287

288

289

290

291

292

293

294

295

296

297

298

299

300

301

302

303

304

305

306

307

308

309

Race, sex, and alcohol use were also associated with changes in the abundance of specific taxa (Fig. 4, Table 5); here, we focus only on the most statistically significant associations (Qvalue $<0.05)$. Relative to non-whites, whites had higher levels of Holdemania and lower levels of Megamonas (Table 5). Interestingly, although Holdemania is one of the 30 most abundant genera in the gut microbiome of individuals from high-income countries (Arumugam et al., 2011), it was absent in a recent study of children from Bangladesh. It is not clear why race might be associated with the abundance of this common but not universal bacterial genus. Megamonas species are associated with colon cancer (Weir et al., 2013), but they are also associated with normal glucose tolerance (vs type 2 diabetes) (Zhang et al., 2013). Again, the connection between Megamonas and race is unclear. Finally, current alcohol users had lower levels of the family Rikenellaceae, which is also depleted in end-stage liver disease (Bajaj et al., 2014), and higher levels of Christensenellaceae (Table 5), a bacterial family enriched in individuals with low BMI (Goodrich et al., 2014). As is true for race, the mechanisms linking sex and alcohol use with changes in these taxa are not yet clear. We were unable to identify specific taxa associated with tobacco use after FDR control.

\section{Discussion}

In this study, we present the MWRP, a reference cohort available for researchers seeking to identify gut microbiome biomarkers related to health or disease. This cohort encompasses a wider range in age and BMI than traditional "healthy" cohorts and contains data on important demographic and health behavior-related variables, such as sex, race, tobacco use, and alcohol use. Consistent with previous studies, we observed large intersubject variability in taxonomic abundance and a small number of core taxa in this cohort (Huse et al., 2012; Human Microbiome Project Consortium, 2012). Moreover, our finding that most of the demographic and health behavior-related variables we investigated were associated with changes in the gut microbiota underscores the importance of having such a diverse cohort available for study. The MWRP can 
310 be used to reduce confounding and increase reproducibility in future studies of the gut 311 microbiome that take place in the Midwestern United States.

312 Within the "normal" cohort of the MWRP, potentially important associations between 313 demographic, health, and behavioral variables and the microbiome emerged. We found an 314 increase in Firmicutes and a decrease in Bacteroidetes in obese subjects; moreover, BMI was 315 associated with decreased species richness as well as overall microbiota structure, confirming the 316 results of previous studies on this subject (Ley et al., 2006; Turnbaugh et al., 2006; 2009;

317 Verdam et al., 2013; Sepp et al., 2014). Health-related behaviors, such as alcohol and tobacco 318 use, are difficult to measure, so they are infrequently studied. Although less attention has been 319 paid to these factors, they nonetheless have been linked to profound changes in the fecal microbiota at the genus level (Biedermann et al., 2013; Mutlu et al., 2014). In our study, alcohol use was associated with overall microbiota structure, and a trend between alcohol use and increased species richness was also detected. Interestingly, alcohol use was associated with an increase in bacteria of the Rikenellaceae family, which are depleted in end-stage liver disease (Bajaj et al., 2014), a potential consequence of alcoholism. Some evidence of association between tobacco use and overall microbiome structure was also observed; given a larger sample size or more detailed data on smoking habits, a significant association between the two may emerge. Many previous studies have found a significant effect of age on the gut microbiota (Hopkins, Sharp \& Macfarlane, 2001; Hébuterne, 2003; Mäkivuokko et al., 2010). However, we did not find an association between age and $\alpha$ - or $\beta$-diversity measures in this study.

The differences in associations between demographic and health behavior-related variables and weighted and unweighted UniFrac distances are potentially meaningful. The unweighted UniFrac distance measures the presence or absence of particular bacterial taxa and is a more qualitative measure of $\beta$-diversity than the weighted UniFrac. The unweighted UniFrac is more sensitive to rare species within a microbial community, as a species will appear absent when its abundance falls below the detection limit of the sequencing machine (Chen et al., 2012). As such, a more significant association with unweighted UniFrac distance (as was found for BMI, sex, alcohol use, race, and tobacco use) may indicate that changes in rare bacteria are altering the community structure. This assertion is supported by our $\alpha$-diversity analysis; demographic and health behavior-related variables were more significantly associated with community richness, 
340 which is based on presence/absence data for OTUs, than overall diversity based on abundance 341 data (i.e., the Shannon index). Finally, in this study, the majority of taxa associated with these 342 variables were rare. Taken together, the results of these separate, yet interrelated, diversity 343 analyses indicate that demographic and health behavior-related factors may affect primarily rare 344 bacterial species within the gut. However, BMI and sex show significant or marginally 345 significant associations with the weighted UniFrac, which is more sensitive to abundant lineages, 346 indicating that some abundant bacterial lineages may also be affected by these factors.

347 Regardless of the analytic tool used, our data suggest that microbial communities are altered by 348 demographic and health behavior-related factors.

The implications of the extensive associations identified in this study are threefold. First, demographic and health behavior-related factors may confound comparative analyses if their distribution differs between groups of interest. These variables must either be matched across groups or adjusted for in statistical models. Importantly, statistical adjustment becomes insufficient when the range of variables differs between groups, as statistical models are less good at extrapolation than interpolation. Nonlinear effects can also impede statistical adjustment. In these cases, sample matching is more appropriate for reducing potential confounding. The MWRP thus provides a good reference panel with which to perform sample matching based on demographic and health behavior-related factors, as it encompasses a wide range for each variable. Second, even if demographic factors do not confound an analysis (i.e., the distribution of these factors is similar between groups), adjustment for these independent predictors in a model will improve statistical power by reducing random error. In some scenarios, significant associations may only be revealed when demographic factors are accounted for. Lastly, other factors important in the Midwest that are difficult to measure in a study like this one, such as dietary habits, may exist. If not well controlled, a case-control-based microbiome biomarker study may capture associations due to these unappreciated confounders, reducing reproducibility. By providing a representative reference sample drawn from the same geographic region that cases are drawn from, the MWRP will be instrumental in minimizing interference by these potential hidden confounders in studies of the microbiome that take place in the Midwest. diverse labs for various conditions, such as BMI, our research has identified frailties that need 
370 further attention if we are to achieve clinical-grade results in microbiome studies. Despite

371 standardized protocols, the implementation of robotic equipment, and uniform technical support,

372 we found evidence of batch effects in this study. We adjusted batch effects in the statistical

373 model to improve statistical power as well as to reduce potential confounding effects (Leek et al.,

374 2010). When batch effects confound a variable of interest, much power will be lost due to batch

375 adjustment. In this case, we suspect that batch effects, which were entangled with age, may have

376 prevented us from detecting some of the associations between age and the microbiome that have

377 been reported in other studies. The clinical utility of microbiome sequencing data may be

378 constrained unless this effect can be overcome. To minimize the impact of batch effects, we

379 advise sample randomization, rigorous and standardized collection methods, and the

380 coordination of sample processing and sequencing for research and biomarker development. For

381 clinical sample management, we propose standardized collection and processing protocols,

382 robotics where applicable, and internal controls.

383

384

385

386

387

388

389

390

391

392

393

394

395

396

397

398

The MWRP also allows us to estimate effect sizes for demographic and health behaviorrelated factors, which is fundamental to moving forward with human microbiome studies that use the stool to study the gut microbiome. It is important to consider factors with larger effect sizes in the study design phase, whereas factors with smaller effect sizes are of less concern. Effect sizes can help prioritize factors when matching subjects from a disease group with samples from a control group. Although $\beta$-diversity association P-values may reflect effect sizes, the coefficient of determination, $\mathrm{R}^{2}$ (i.e., the percent of variation in an outcome variable explained by a factor of interest), is a more suitable measure (McArdle \& Anderson, 2001). Using unweighted UniFrac distances to summarize overall microbiota variability, adjusted $\mathrm{R}^{2}$ values were $0.95 \%$ for race, $0.88 \%$ for BMI, $0.53 \%$ for alcohol use, $0.46 \%$ for sex, $0.33 \%$ for tobacco use, and $0.0 \%$ for age. The small values of $\mathrm{R}^{2}$ may be partially due to noise from sample collection, preparation, and sequencing, but they also indicate that these factors explain only a small fraction of the large intersubject variability of the gut microbiota. Although small in effect size, these factors may still be important confounders, as many of the associations we find in public health also have very small effect sizes. Race and BMI, which have the largest $\mathrm{R}^{2}$ values, warrant special attention in study design. 
The diverse associations between demographic and health behavior-related factors and

400

401

402

403

404

405

406

407

408

409

410

411

412

413

414

415

416

417

418

419

420

421

422

423

424

425

426

427

428

429

430 the gut microbiota, and their small effect sizes, have motivated us to expand the list of demographic and behavioral variables investigated in the future, with the goal of identifying more factors that shape the gut microbiota; indeed, our next step is to expand the MWRP sample to explore factors such as diet, exercise, depression, and anxiety. A larger sample, coupled with more detailed documentation of variables, will be instrumental for addressing potential confounding due to factors that are rarely represented in the normal population but are enriched in groups with diseases, as well as for identifying the microbiota features that best distinguish between diseased and healthy states. In addition, we will extend our current taxonomic profiling to functional profiling, to characterize a possible functional core in the MWRP. Longitudinal studies of the microbiome, which are conducive to establishing a mechanistic link between the microbiota and a phenotype, will also be pursued. Finally, similar studies of the impact of demographics on microbiomes from other body sites will be conducted to facilitate microbiome biomarker discovery for a broader range of diseases.

\section{Conclusions}

In conclusion, the MWRP will be instrumental in elucidating demographic and health behaviorrelated factors impacting the human gut microbiota, as well as increasing reproducibility in microbiome biomarker discovery. It represents a key step in translating microbiome discoveries into clinical applications and, ultimately, in improving patient care.

Abreu MT, Peek RM 2014. Gastrointestinal Malignancy and the Microbiome. Gastroenterology 146:1534-1546.e3.

Arumugam M, Raes J, Pelletier E, Le Paslier D, Yamada T, Mende DR, Fernandes GR, Tap J, Bruls T, Batto J-M, Bertalan M, Borruel N, Casellas F, Fernandez L, Gautier L, Hansen T, Hattori M, Hayashi T, Kleerebezem M, Kurokawa K, Leclerc M, Levenez F, Manichanh C, Nielsen HB, Nielsen T, Pons N, Poulain J, Qin J, Sicheritz-Ponten T, Tims S, Torrents D, Ugarte E, Zoetendal EG, Wang J, Guarner F, Pedersen O, de Vos WM, Brunak S, Doré J, additional members MC, Weissenbach J, Ehrlich SD, Bork P 2011. Enterotypes of the human gut microbiome. Nature 473:174-180.

Bajaj JS, Heuman DM, Hylemon PB, Sanyal AJ, White MB, Monteith P, Noble NA, Unser AB, Daita K, Fisher AR, Sikaroodi M, Gillevet PM 2014. Altered profile of human gut 
431

432

433

434

435

436

437

438

439

440

441

442

443

444

445

446

447

448

449

450

451

452

453

454

455

456

457

458

459

460

461

462

463

464

465

466

467

468

469

470

471

472

473

474

475

476 microbiome is associated with cirrhosis and its complications. Journal of Hepatology 60:940-947.

Biedermann L, Zeitz J, Mwinyi J, Sutter-Minder E, Rehman A, Ott SJ, Steurer-Stey C, Frei A, Frei P, Scharl M, Loessner MJ, Vavricka SR, Fried M, Schreiber S, Schuppler M, Rogler G 2013. Smoking Cessation Induces Profound Changes in the Composition of the Intestinal Microbiota in Humans. PloS one 8:e59260.

Cani P, Delzenne N 2009. The Role of the Gut Microbiota in Energy Metabolism and Metabolic Disease. Current Pharmaceutical Design 15:1546-1558.

Caporaso JG, Lauber CL, Walters WA, Berg-Lyons D, Huntley J, Fierer N, Owens SM, Betley J, Fraser L, Bauer M, Gormley N, Gilbert JA, Smith G, Knight R 2012. Ultra-high-throughput microbial community analysis on the Illumina HiSeq and MiSeq platforms. The ISME journal 6:1621-1624.

Chao A, Gotelli NJ, Hsieh TC, Sander EL, Ma KH, Colwell RK, Ellison AM 2014. Rarefaction and extrapolation with Hill numbers: a framework for sampling and estimation in species diversity studies. Ecological Monographs 84:45-67.

Chen J, Bittinger K, Charlson ES, Hoffmann C, Lewis J, Wu GD, Collman RG, Bushman FD, Li $\mathrm{H}$ 2012. Associating microbiome composition with environmental covariates using generalized UniFrac distances. Bioinformatics 28:2106-2113.

Chiu C-H, Chao A 2015. Estimating and comparing microbial diversity in the presence of sequencing errors. PeerJ Preprint. https://peerj.com/preprints/1353.pdf

Cho I, Blaser MJ 2012. The human microbiome: at the interface of health and disease. Nature Reviews Genetics 13:260-270.

David R 2013. Microbiome: A bacterial trigger for liver cancer. Nature reviews. Microbiology 11:509-509.

Flores GE, Caporaso JG, Henley JB, Rideout JR, Domogala D, Chase J, Leff JW, VázquezBaeza Y, Gonzalez A, Knight R, Dunn RR, Fierer N 2014. Temporal variability is a personalized feature of the human microbiome. Genome Biology 15:531.

Foxman B, Rosenthal M 2013. Implications of the human microbiome project for epidemiology. American journal of epidemiology 177:197-201.

Gevers D, Knight R, Petrosino JF, Huang K, McGuire AL, Birren BW, Nelson KE, White O, Methé BA, Huttenhower C 2012. The human microbiome project: a community resource for the healthy human microbiome. PLoS biology 10:e1001377.

Goodrich JK, Waters JL, Poole AC, Sutter JL, Koren O, Blekhman R, Beaumont M, Van Treuren W, Knight R, Bell JT, Spector TD, Clark AG, Ley RE 2014. Human Genetics Shape the Gut Microbiome. Cell 159:789-799.

Hébuterne X 2003. Gut changes attributed to ageing: effects on intestinal microflora. Current Opinion in Clinical Nutrition \& Metabolic Care 6:49.

Hopkins MJ, Sharp R, Macfarlane GT 2001. Age and disease related changes in intestinal bacterial populations assessed by cell culture, 16S rRNA abundance, and community cellular fatty acid profiles. Gut 48:198-205.

Human Microbiome Project Consortium 2012. Structure, function and diversity of the healthy human microbiome. Nature 486:207-214.

Huse SM, Ye Y, Zhou Y, Fodor AA 2012. A core human microbiome as viewed through 16S rRNA sequence clusters. PloS one 7:e34242.

Jeraldo P, Kalari K, Chen X, Bhavsar J, Mangalam A, White B, Nelson H, Kocher J-P, Chia N 2014. IM-TORNADO: A Tool for Comparison of 16S Reads from Paired-End Libraries. 
PloS one 9:e114804.

Leek JT, Scharpf RB, Bravo HC, Simcha D, Langmead B, Johnson WE, Geman D, Baggerly K, Irizarry RA 2010. Tackling the widespread and critical impact of batch effects in highthroughput data. Nature Reviews Genetics 11:733-739.

Ley RE, Turnbaugh PJ, Klein S, Gordon JI 2006. Microbial ecology: Human gut microbes associated with obesity. Nature 444:1022-1023.

Lozupone C, Knight R 2005. UniFrac: a new phylogenetic method for comparing microbial communities. Applied and Environmental Microbiology 71:8228-8235.

Mäkivuokko H, Tiihonen K, Tynkkynen S, Paulin L, Rautonen N 2010. The effect of age and non-steroidal anti-inflammatory drugs on human intestinal microbiota composition. The British journal of nutrition 103:227-234.

McArdle BH, Anderson MJ 2001. Fitting Multivariate Models to Community Data: A Comment on Distance-Based Redundancy Analysis. Ecology 82:290-297.

Mutlu EA, Keshavarzian A, Losurdo J, Swanson G, Siewe B, Forsyth C, French A, DeMarais P, Sun Y, Koenig L, Cox S, Engen P, Chakradeo P, Abbasi R, Gorenz A, Burns C, Landay A 2014. A Compositional Look at the Human Gastrointestinal Microbiome and Immune Activation Parameters in HIV Infected Subjects. PLOS Pathogen 10:e1003829.

Olson JE, Ryu E, Johnson KJ, Koenig BA, Maschke KJ, Morrisette JA, Liebow M, Takahashi PY, Fredericksen ZS, Sharma RG, Anderson KS, Hathcock MA, Carnahan JA, Pathak J, Lindor NM, Beebe TJ, Thibodeau SN, Cerhan JR 2013. The Mayo Clinic Biobank: a building block for individualized medicine. Mayo Clinic proceedings 88:952-962.

Qin J, Li R, Raes J, Arumugam M, Burgdorf KS, Manichanh C, Nielsen T, Pons N, Levenez F, Yamada T, Mende DR, Li J, Xu J, Li S, Li D, Cao J, Wang B, Liang H, Zheng H, Xie Y, Tap J, Lepage P, Bertalan M, Batto J-M, Hansen T, Le Paslier D, Linneberg A, Nielsen HB, Pelletier E, Renault P, Sicheritz-Ponten T, Turner K, Zhu H, Yu C, Li S, Jian M, Zhou Y, Li Y, Zhang X, Li S, Qin N, Yang H, Wang J, Brunak S, Doré J, Guarner F, Kristiansen K, Pedersen O, Parkhill J, Weissenbach J, Antolin M, Artiguenave F, Blottiere H, Borruel N, Bruls T, Casellas F, Chervaux C, Cultrone A, Delorme C, Denariaz G, Dervyn R, Forte M, Friss C, van de Guchte M, Guedon E, Haimet F, Jamet A, Juste C, Kaci G, Kleerebezem M, Knol J, Kristensen M, Layec S, Le Roux K, Leclerc M, Maguin E, Minardi RM, Oozeer R, Rescigno M, Sanchez N, Tims S, Torrejon T, Varela E, de Vos W, Winogradsky Y, Zoetendal E, Bork P, Ehrlich SD, Wang J 2010. A human gut microbial gene catalogue established by metagenomic sequencing. Nature 464:59-65.

Qin J, Li Y, Cai Z, Li S, Zhu J, Zhang F, Liang S, Zhang W, Guan Y, Shen D, Peng Y, Zhang D, Jie Z, Wu W, Qin Y, Xue W, Li J, Han L, Lu D, Wu P, Dai Y, Sun X, Li Z, Tang A, Zhong S, Li X, Chen W, Xu R, Wang M, Feng Q, Gong M, Yu J, Zhang Y, Zhang M, Hansen T, Sanchez G, Raes J, Falony G, Okuda S, Almeida M, LeChatelier E, Renault P, Pons N, Batto J-M, Zhang Z, Chen H, Yang R, Zheng W, Li S, Yang H, Wang J, Ehrlich SD, Nielsen R, Pedersen O, Kristiansen K, Wang J 2012. A metagenome-wide association study of gut microbiota in type 2 diabetes. Nature 490:55-60.

Round JL, Mazmanian SK 2009. The gut microbiota shapes intestinal immune responses during health and disease. Nature Reviews Immunology 9:313-323.

Rubinstein MR, Wang X, Liu W, Hao Y, Cai G, Han YW 2014. Fusobacterium nucleatum Promotes Colorectal Carcinogenesis by Modulating E-Cadherin/ $\beta$-Catenin Signaling via its FadA Adhesin. Cell host \& microbe 14:195-206.

Sartor RB 2004. Therapeutic manipulation of the enteric microflora in inflammatory bowel 
diseases: antibiotics, probiotics, and prebiotics. Gastroenterology 126:1620-1633.

Scher JU, Sczesnak A, Longman RS, Segata N, Ubeda C, Bielski C, Rostron T, Cerundolo V, Pamer EG, Abramson SB, Huttenhower C, Littman DR 2013. Expansion of intestinal Prevotella copri correlates with enhanced susceptibility to arthritis. eLife 2:e01202-e01202. Schubert AM, Rogers MAM, Ring C, Mogle J, Petrosino JP, Young VB, Aronoff DM, Schloss PD 2014. Microbiome data distinguish patients with Clostridium difficile infection and nonC. difficile-associated diarrhea from healthy controls. mBio 5:e01021-14.

Sepp E, Kolk H, Lõivukene K, Mikelsaar M 2014. Higher blood glucose level associated with body mass index and gut microbiota in elderly people. Microbial Ecology in Health and Disease 25:2391.

Turnbaugh PJ, Hamady M, Yatsunenko T, Cantarel BL, Duncan A, Ley RE, Sogin ML, Jones WJ, Roe BA, Affourtit JP, Egholm M, Henrissat B, Heath AC, Knight R, Gordon JI 2009. A core gut microbiome in obese and lean twins. Nature 457:480-484.

Turnbaugh PJ, Ley RE, Mahowald MA, Magrini V, Mardis ER, Gordon JI 2006. An obesityassociated gut microbiome with increased capacity for energy harvest. Nature 444:1027131.

Verdam FJ, Fuentes S, de Jonge C, Zoetendal EG, Erbil R, Greve JW, Buurman WA, de Vos WM, Rensen SS 2013. Human intestinal microbiota composition is associated with local and systemic inflammation in obesity. Obesity 21:E607-E615.

Walters WA, Xu Z, Knight R 2014. Meta-analyses of human gut microbes associated with obesity and IBD. FEBS Letters 588:4223-4233.

Weir TL, Manter DK, Sheflin AM, Barnett BA, Heuberger AL, Ryan EP 2013. Stool Microbiome and Metabolome Differences between Colorectal Cancer Patients and Healthy Adults. PloS one 8:e70803.

Zhang J, Guo Z, Xue Z, Sun Z, Zhang M, Wang L, Wang G, Wang F, Xu J, Cao H, Xu H, Lv Q, Zhong Z, Chen Y, Qimuge S, Menghe B, Zheng Y, Zhao L, Chen W, Zhang H 2015. A phylo-functional core of gut microbiota in healthy young Chinese cohorts across lifestyles, geography and ethnicities. The ISME journal 9:1979-1990.

Zhang X, Shen D, Fang Z, Jie Z, Qiu X, Zhang C, Chen Y, Ji L 2013. Human Gut Microbiota Changes Reveal the Progression of Glucose Intolerance. PloS one 8:e71108. 
Figure 1 (on next page)

Gut microbiota profile of the MWRP.

A) Distribution of OTU abundance. B) Distribution of OTU prevalence. C) Relative abundance of major bacteria at the level of the phylum. D) Relative abundance of major bacteria at the level of the family. 
A

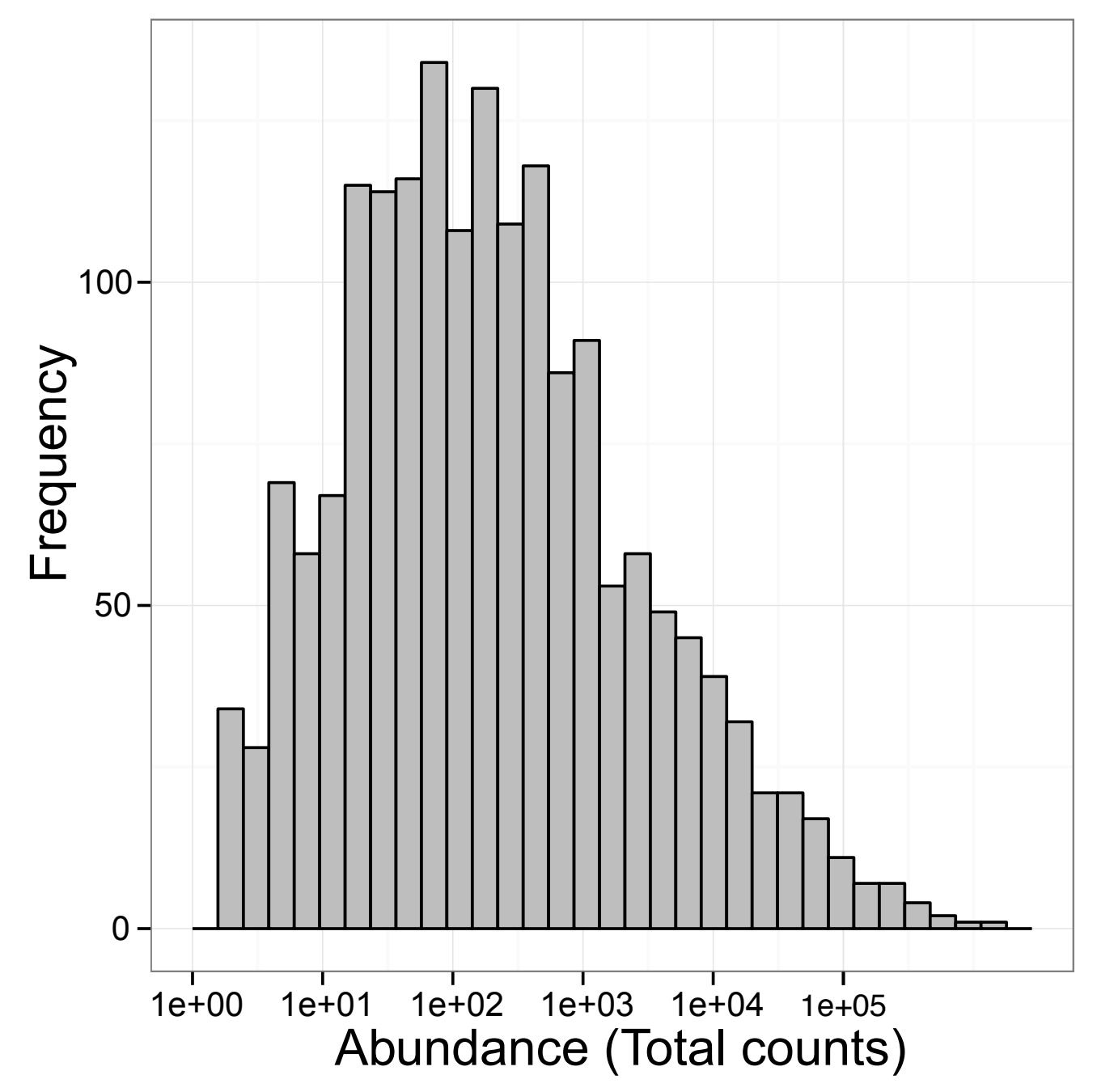

C

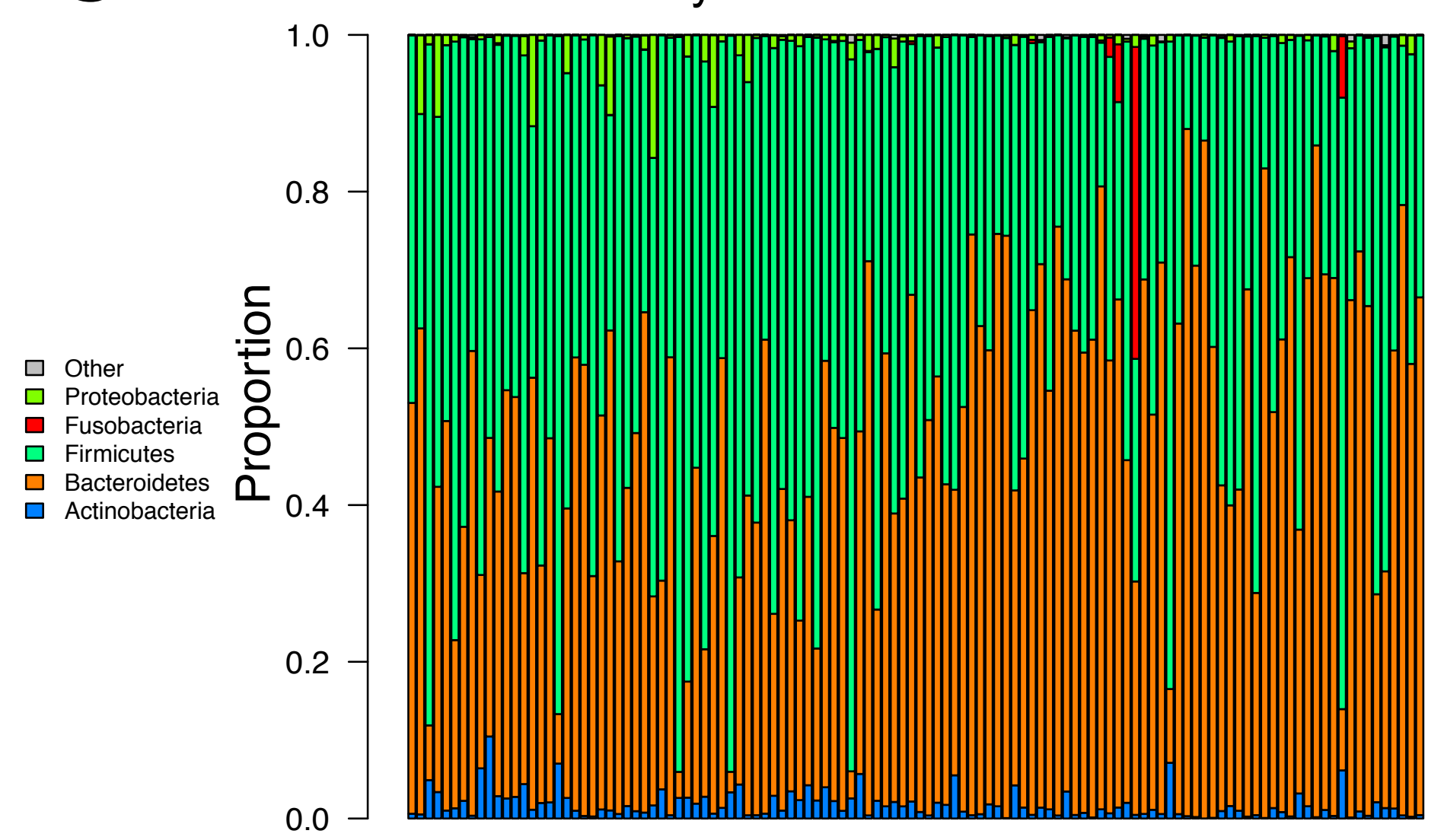

Manuscript to be reviewed

B

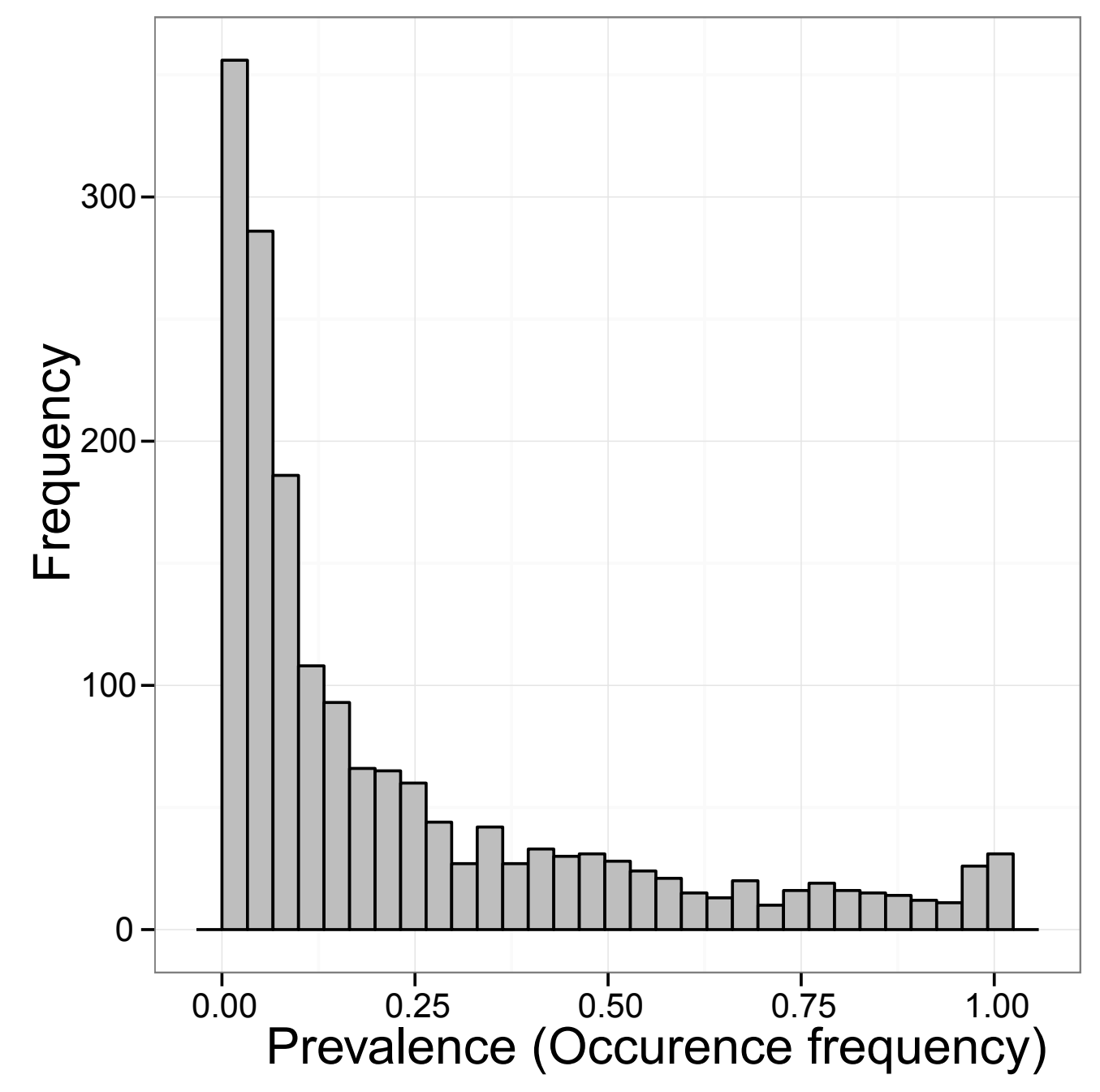

D

$\square$ Other
$\square$ Proteobacteria;Enterobacteriaceae ㅁ. Firmicutes;Veillonellaceae
다micutes;Streptococcaceae

ㅁ Firmicutes;Ruminococcaceae $\square$ Firmicutes;Peptostreptococcaceae ㅁ Firmicutes;Lachnospiraceae 을 0.6 ․ Firmicutes;Erysipelotrichace - Firmicutes;Clostridiaceae $\mathrm{Chritensenellaceae}$ - Bacteroidetes;S24-7 ㅁ Bacteroidetes:Rikenellaceae - Bacteroidetes;Porphyromonadacea - Bacteridetes:Odoribacteraceae $\square$ Bacteroidetes;Barnesiellaceae

ㅁ. Bacteroidetes;Bacteroidaceae
․ Actinobacteria;Coriobacteriaceae

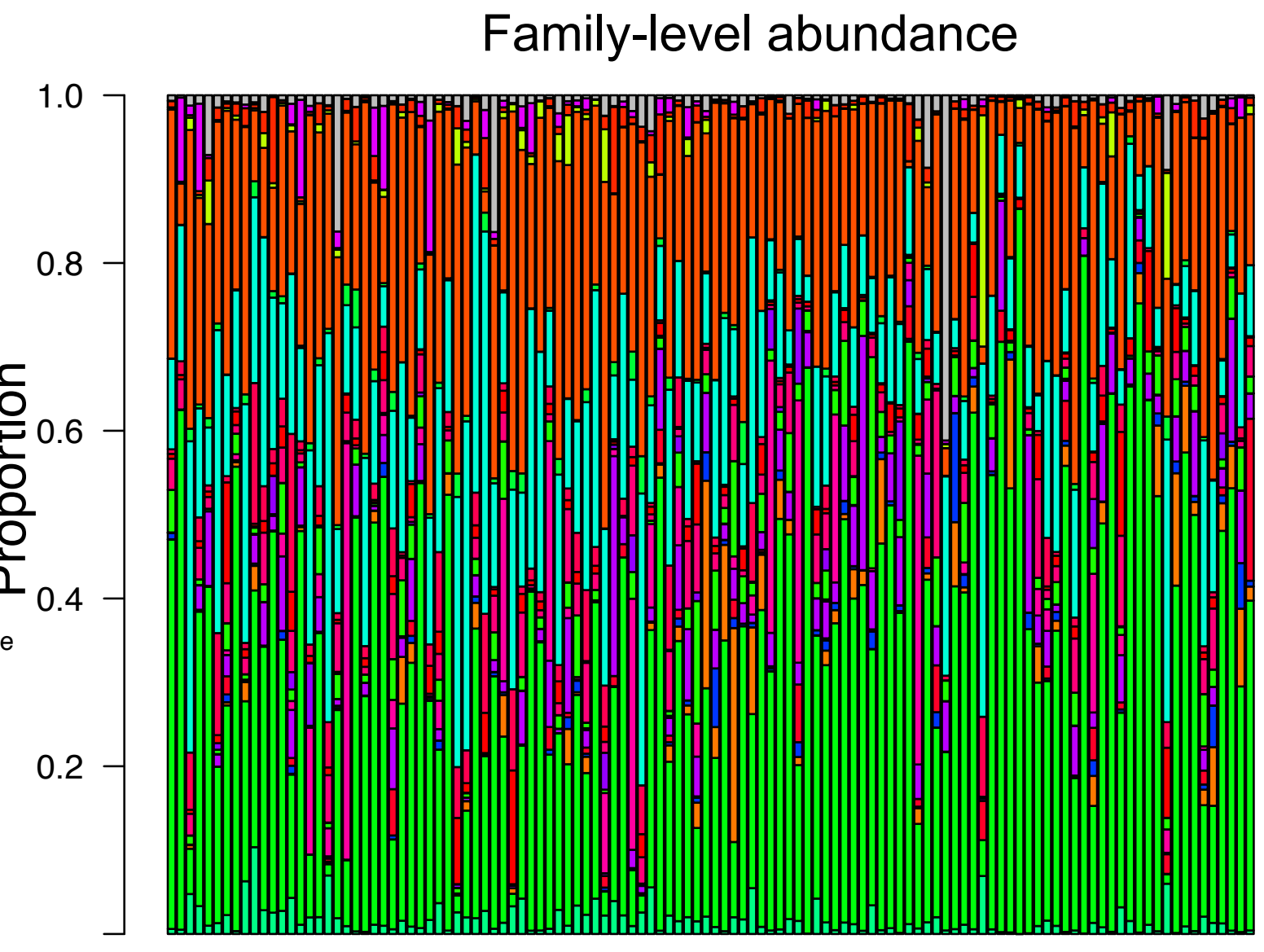




\section{Figure 2 (on next page)}

Associations between demographic and health behavior-related factors and the overall gut microbiota structure.

A) Increased BMI is associated with decreased species richness (i.e., the observed number of OTUs). B) Increased species richness was observed in white subjects. The three horizontal lines of the box represent the first, second (median), and third quartiles, respectively, with the whisk extending to the 1.5 interquartile range (IQR). C) Principal coordinate analysis (PCOA) plot showing a sex effect. D) PCoA plot showing an alcohol effect. Samples are colored according to group membership, and plotted on axes corresponding the first two principal coordinates (PCs). The percentage of variability explained by each PC is indicated in the parentheses. 
A

BNiirerffect

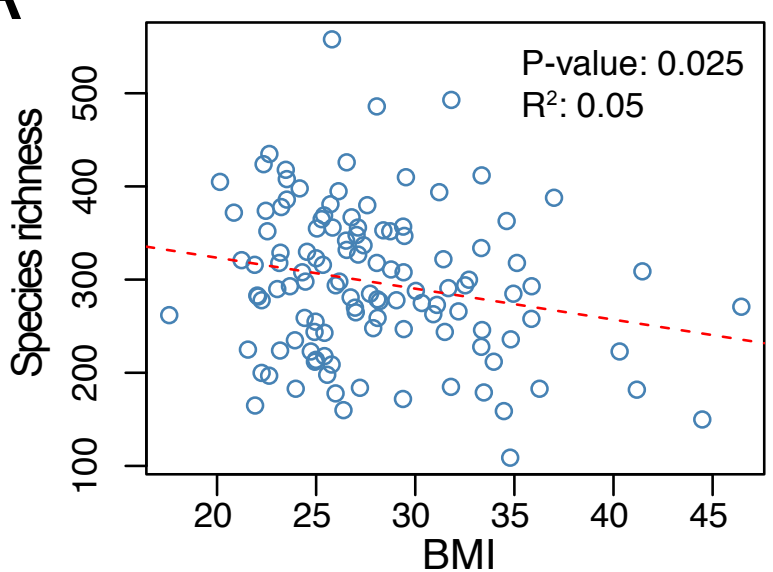

C

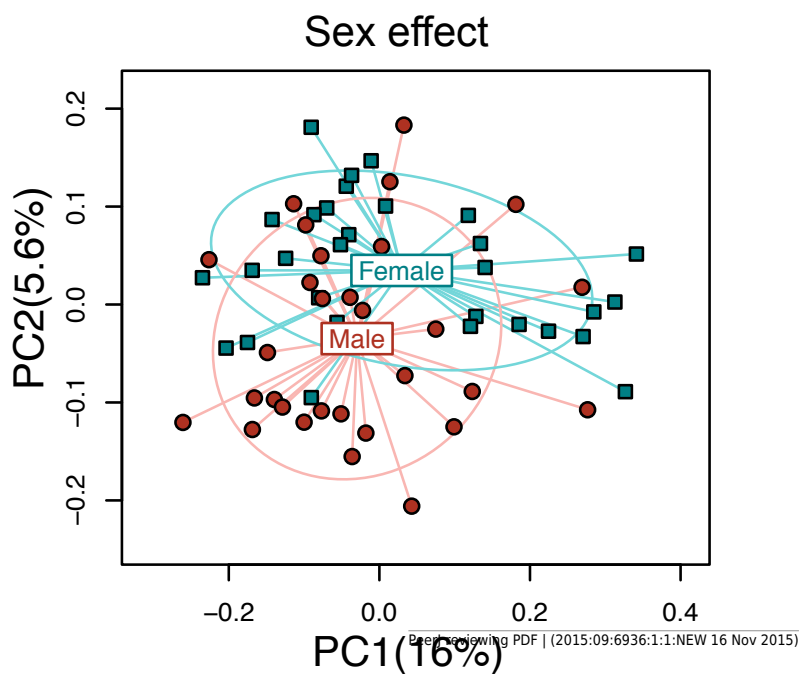

Manuscript to be reviRace effect

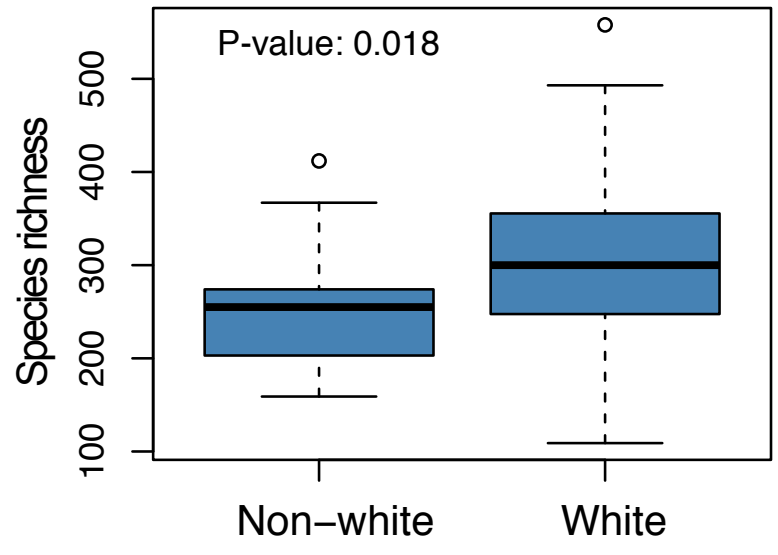

D

Alcohol effect

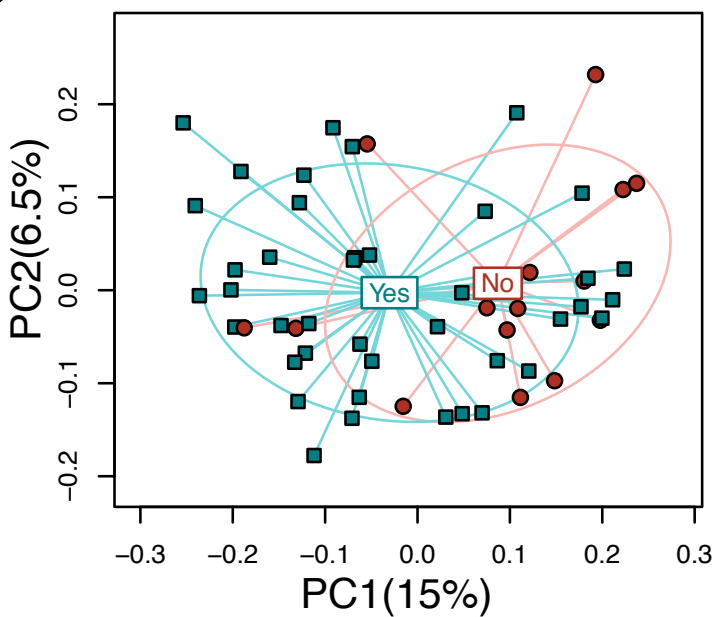




\section{Figure 3 (on next page)}

Percentage of variability explained by the demographic and health behavior-related factors.

The unweighted UniFrac distance was used to summarize the microbiota variability. The distance-based $R^{2}$ was adjusted to reduce inflation due to a small sample size. 


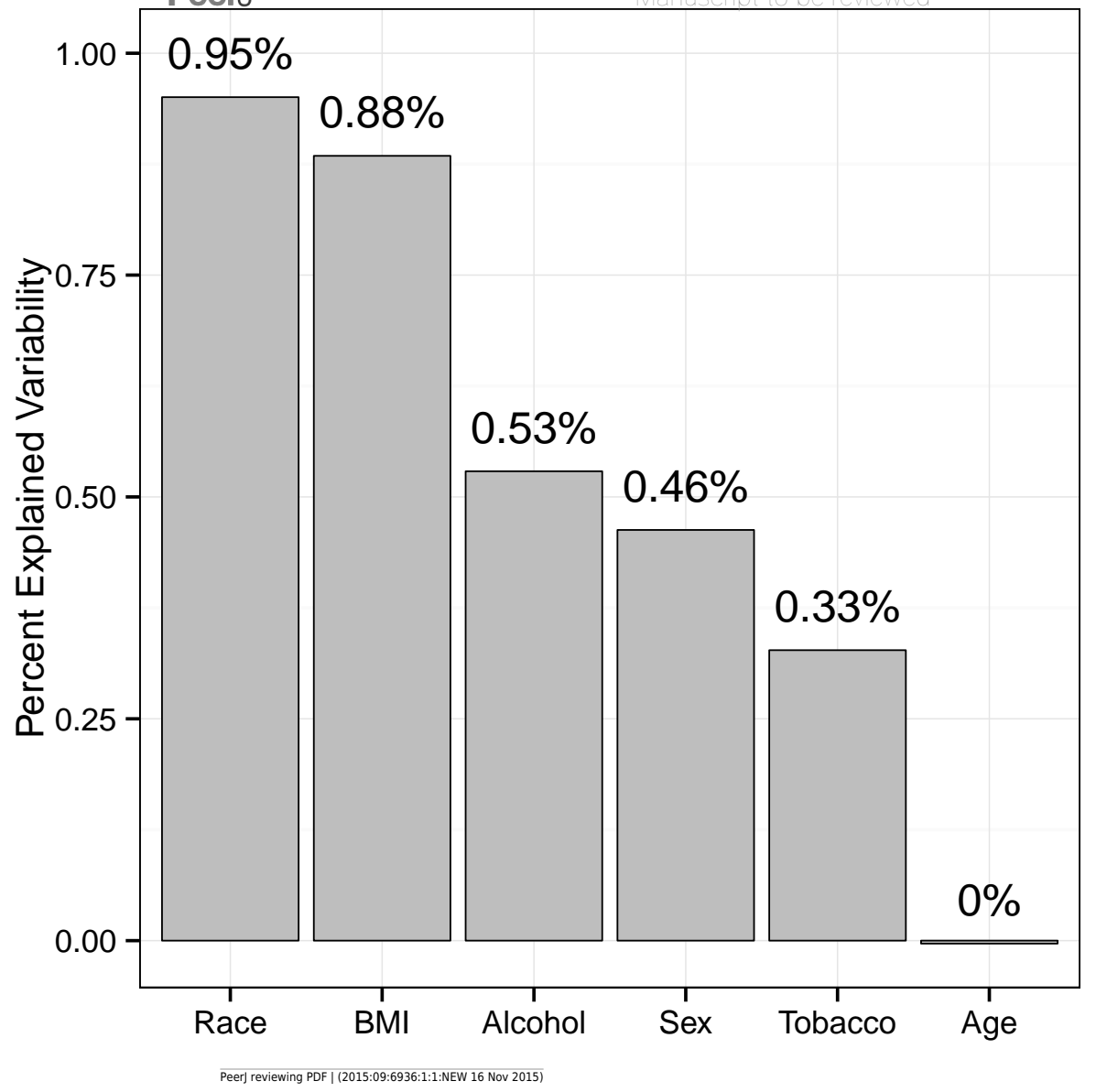


Figure 4 (on next page)

Microbial signatures of demographic and health behavior-related factors: BMI, race, sex, and alcohol use.

Barplots show the mean relative abundance and standard error of bacterial taxa in each subject subgroup. Taxa were selected based on univariate association tests with an FDR of $10 \%$. Here, we have discretized BMI into normal and obese groups based on the cutoff of 30 $\mathrm{kg} / \mathrm{m}^{2}$ for visualization purposes. However, we treated BMI as a continuous outcome in our association tests. 
BMI

Normal Obese

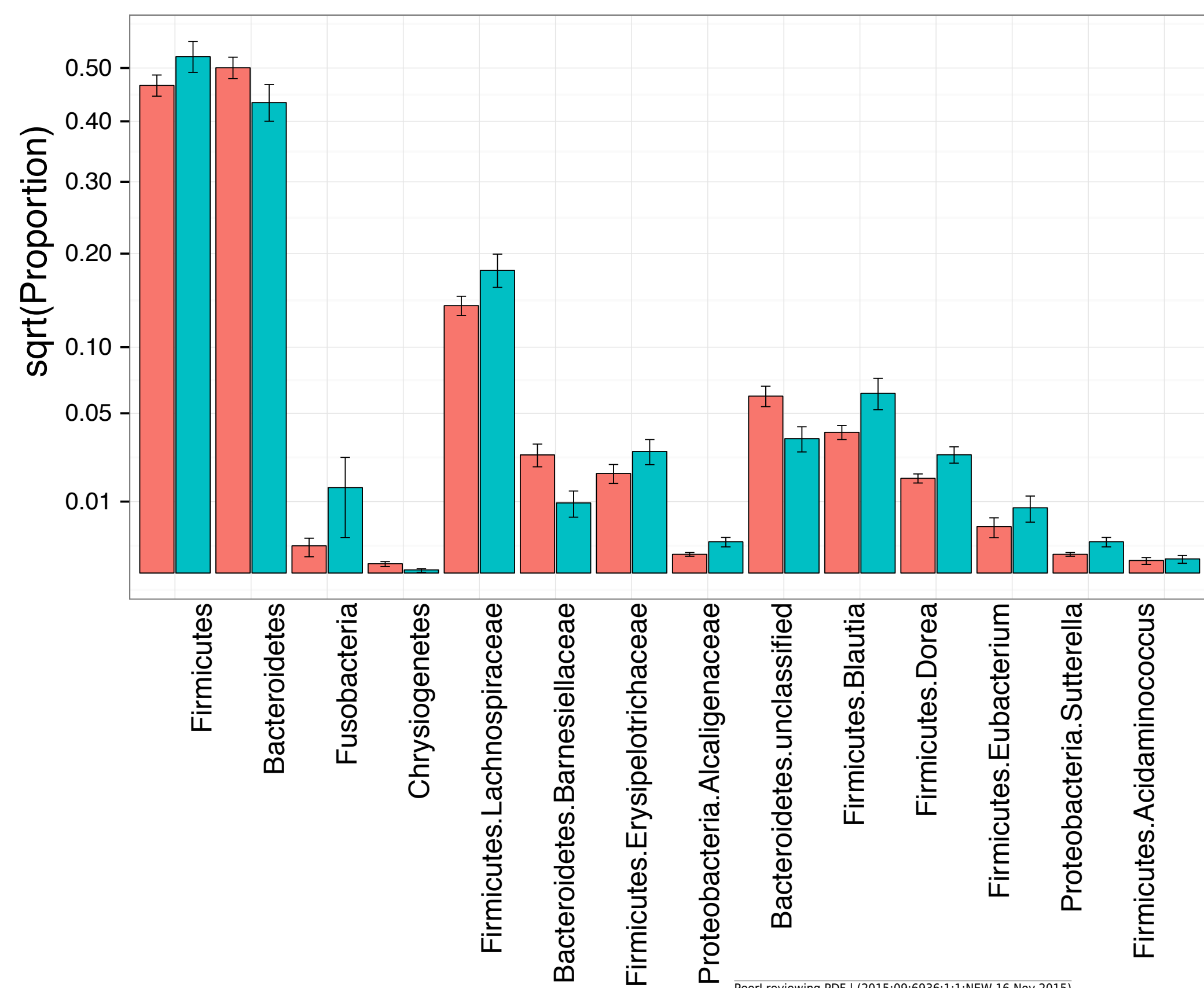

Race

Non-white White

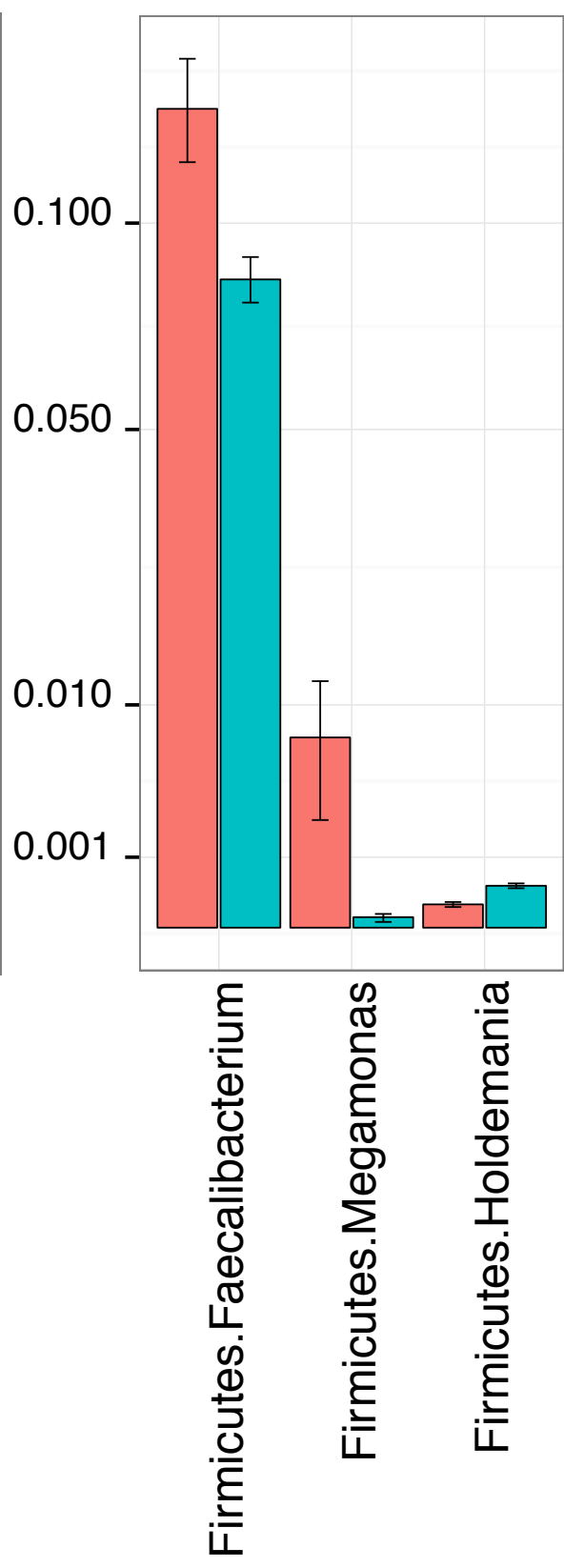

Sex

Male Female

Alcohol

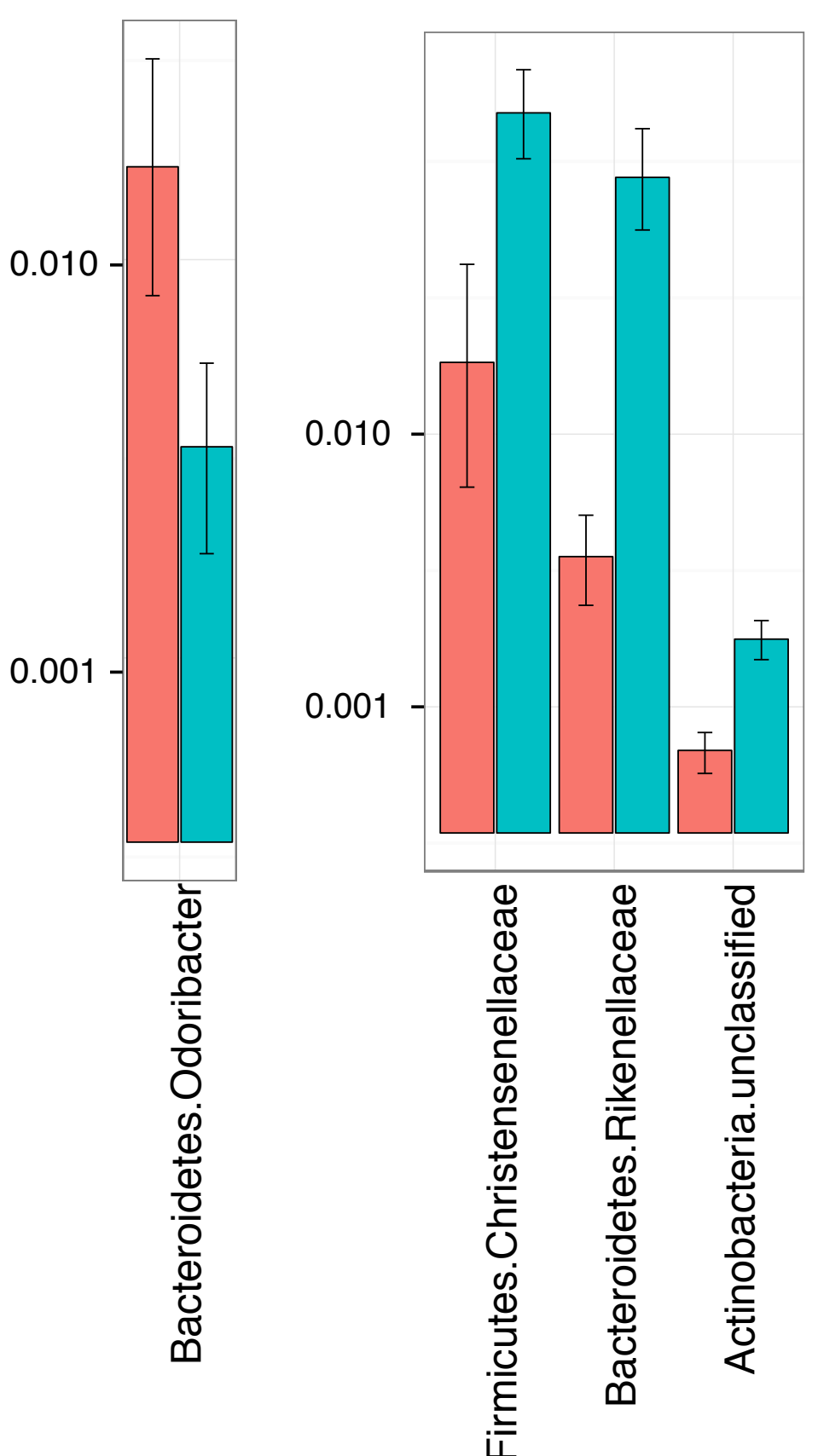




\section{Table $\mathbf{1}$ (on next page)}

Comparison between subjects in the MWRP $(\mathrm{N}=118)$ and the BRFSS cohort on the major demographic and health behavior-related factors. 


\begin{tabular}{|c|c|c|c|c|c|}
\hline & & \multicolumn{2}{|c|}{ MWRP } & \multirow{2}{*}{$\begin{array}{c}\text { BRFSS } \\
\%\end{array}$} & \multirow[b]{2}{*}{ P-value } \\
\hline & & Number & $\%$ & & \\
\hline \multirow{5}{*}{$\begin{array}{c}\text { Age }<50 \\
\text { years } \\
(n=58)\end{array}$} & Sex, F & 30 & 51.7 & 49.3 & 0.81 \\
\hline & Race, W & 49 & 84.5 & 73.5 & 0.08 \\
\hline & $\mathrm{BMI}>30 \mathrm{~kg} / \mathrm{m}^{2}$ & 14 & 24.1 & 25.6 & 0.92 \\
\hline & Alcohol use (Y) & 41 & 70.7 & 60.2 & 0.13 \\
\hline & Smoking (Y) & 9 & 15.5 & 23.3 & 0.21 \\
\hline \multirow{5}{*}{$\begin{array}{l}\text { Age } \geq 50 \\
\text { years } \\
(\mathrm{n}=60)\end{array}$} & Sex, F & 30 & 50.0 & 53.9 & 0.63 \\
\hline & Race, W & 54 & 90.0 & 83.2 & 0.22 \\
\hline & $\mathrm{BMI}>30 \mathrm{~kg} / \mathrm{m}^{2}$ & 20 & 33.3 & 29.5 & 0.61 \\
\hline & Alcohol use (Y) & 39 & 65.0 & 48.4 & 0.02 \\
\hline & Smoking (Y) & 8 & 13.3 & 15.7 & 0.74 \\
\hline
\end{tabular}

1 Abbreviations: BMI, body mass index; BRFSS, Behavioral Risk Factor Surveillance Survey; F, 2 female; MWRP, Midwestern Reference Panel; W, white; Y, yes. 


\section{Table 2 (on next page)}

Most prevalent taxa ( $\geq 95 \%)$ at the phylum, family, and genus level identified in the MWRP. 


\begin{tabular}{|c|c|c|c|}
\hline \multicolumn{4}{|c|}{ Phylum } \\
\hline Actinobacteria & Bacteroidetes & Firmicutes & Proteobacteria \\
\hline \multicolumn{4}{|c|}{ Family } \\
\hline Coriobacteriaceae & Bacteroidaceae & Barnesiellaceae & Porphyromonadaceae \\
\hline Prevotellaceae & Ruminococcaceae & Peptostreptococcaceae & Paraprevotellaceae \\
\hline Rikenellaceae & Clostridiaceae & Erysipelotrichaceae & Lachnospiraceae \\
\hline Streptococcaceae & Veillonellaceae & Christensenellaceae & Mogibacteriaceae \\
\hline Enterobacteriaceae & Odoribacteraceae & & \\
\hline \multicolumn{4}{|c|}{ Genus } \\
\hline Bacteroides & Parabacteroides & Prevotella & Blautia \\
\hline Faecalibacterium & Butyrivibrio & Streptococcus & Erwinia \\
\hline Clostridium & Coprobacillus & Coprococcus & Dorea \\
\hline Oscillospira & Roseburia & Ruminococcus & Shuttleworthia \\
\hline Moryella & Lachnospira & Adlercreutzia & Holdemania \\
\hline Eubacterium & Christensenella & & \\
\hline
\end{tabular}

2 


\section{Table 3(on next page)}

Associations (P-values) between demographic and health behavior-related factors and $\alpha$-diversity measures in the MWRP. 
1

\begin{tabular}{|r|r|r|r|r|r|r|}
\hline & \multicolumn{1}{|c|}{ Age } & \multicolumn{1}{c|}{ Sex } & \multicolumn{1}{c|}{ BMI } & \multicolumn{1}{c|}{ Race } & Tobacco & Alcohol \\
\hline Observed OTUs & 0.463 & 0.062 & $\mathbf{0 . 0 1 7}$ & $\mathbf{0 . 0 1 3}$ & 0.381 & $\mathbf{0 . 0 4 0}$ \\
\hline Shannon index & 0.115 & 0.381 & 0.684 & 0.425 & 0.350 & 0.138 \\
\hline
\end{tabular}

2 Abbreviations: BMI, body mass index; OTU, operational taxonomic unit.

3 
Table 4 (on next page)

Associations (P-values) between demographic and health behavior-related factors and $\beta$-diversity measures in the MWRP. 
1

\begin{tabular}{|l|l|l|l|l|l|l|}
\hline & Age & Sex & BMI & Race & Tobacco & Alcohol \\
\hline Unweighted UniFrac & 0.351 & $\mathbf{0 . 0 4 9}$ & $\mathbf{0 . 0 0 6}$ & $\mathbf{0 . 0 0 3}$ & 0.091 & $\mathbf{0 . 0 4 1}$ \\
\hline Weighted UniFrac & 0.776 & 0.073 & $\mathbf{0 . 0 3 0}$ & 0.227 & 0.143 & 0.466 \\
\hline
\end{tabular}

2 Abbreviation: BMI, body mass index.

3

4 


\section{Table 5 (on next page)}

Bacterial taxa associated with $\mathrm{BMI}$, race, sex, and alcohol use based on univariate associations at an FDR of $10 \%$. 


\begin{tabular}{|c|c|c|c|c|}
\hline \multirow[b]{2}{*}{ BMI } & \multicolumn{2}{|c|}{ Mean proportion } & \multirow{2}{*}{$\begin{array}{l}\text { Log2 Fold } \\
\text { Change }\end{array}$} & \multirow{2}{*}{$Q$ value } \\
\hline & Obese & Normal & & \\
\hline \multicolumn{5}{|c|}{ Phylum } \\
\hline Bacteroidetes & 4.339E-01 & $5.004 \mathrm{E}-01$ & -0.206 & 0.080 \\
\hline Firmicutes & $5.226 \mathrm{E}-01$ & 4.660E-01 & 0.165 & 0.080 \\
\hline Chrysiogenetes & 1.927E-05 & $1.680 \mathrm{E}-04$ & -3.124 & 0.080 \\
\hline Fusobacteria & 1.433E-02 & $1.446 \mathrm{E}-03$ & 3.309 & 0.080 \\
\hline \multicolumn{5}{|c|}{ Family } \\
\hline Bacteroidetes;Barnesiellaceae & 9.637E-03 & $2.734 \mathrm{E}-02$ & -1.505 & 0.098 \\
\hline Firmicutes;Erysipelotrichaceae & 2.898E-02 & $1.942 \mathrm{E}-02$ & 0.578 & 0.078 \\
\hline Firmicutes;Lachnospiraceae & 1.796E-01 & $1.401 \mathrm{E}-01$ & 0.358 & 0.098 \\
\hline Proteobacteria;Alcaligenaceae & 1.909E-03 & 6.867E-04 & 1.475 & 0.098 \\
\hline \multicolumn{5}{|c|}{ Genus } \\
\hline Bacteroidetes;unclassified & 3.534E-02 & $6.134 \mathrm{E}-02$ & -0.795 & 0.049 \\
\hline Firmicutes;Acidaminococcus & 3.915E-04 & $3.081 \mathrm{E}-04$ & 0.346 & 0.098 \\
\hline Firmicutes;Blautia & $6.326 \mathrm{E}-02$ & $3.883 E-02$ & 0.704 & 0.049 \\
\hline Firmicutes;Dorea & 2.743E-02 & 1.757E-02 & 0.643 & 0.049 \\
\hline Firmicutes;Eubacterium & 8.339E-03 & 4.210E-03 & 0.986 & 0.049 \\
\hline Proteobacteria;Sutterella & 1.909E-03 & 6.867E-04 & 1.475 & 0.098 \\
\hline & \multicolumn{2}{|c|}{ Mean proportion } & Log2 Fold & مulu \\
\hline Race & Non-white & White & Change & Q value \\
\hline \multicolumn{5}{|c|}{ Genus } \\
\hline Firmicutes;Faecalibacterium & 1.351E-01 & $8.466 \mathrm{E}-02$ & 0.674 & 0.098 \\
\hline Firmicutes;Holdemania & 1.092E-04 & $3.542 \mathrm{E}-04$ & -1.698 & 0.049 \\
\hline Firmicutes;Megamonas & 7.288E-03 & $2.226 \mathrm{E}-05$ & 8.355 & 0.049 \\
\hline \multirow[b]{2}{*}{ Sex } & \multicolumn{2}{|c|}{ Mean proportion } & Log2 Fold & מula \\
\hline & Yes & No & Change & U value \\
\hline \multicolumn{5}{|c|}{ Genus } \\
\hline Bacteroidetes;Odoribacter & $1.344 \mathrm{E}-02$ & 4.606E-03 & 1.545 & 0.067 \\
\hline & \multicolumn{2}{|c|}{ Mean proportion } & Log2 Fold & value \\
\hline Alcohol intake & Yes & No & Change & U value \\
\hline \multicolumn{5}{|c|}{ Family } \\
\hline Bacteroidetes;Rikenellaceae & $2.702 \mathrm{E}-02$ & 4.803E-03 & 2.492 & 0.002 \\
\hline Firmicutes;Christensenellaceae & $3.260 \mathrm{E}-02$ & 1.393E-02 & 1.227 & 0.030 \\
\hline \multicolumn{5}{|c|}{ Genus } \\
\hline Actinobacteria;unclassified & 2.362E-03 & $4.288 \mathrm{E}-04$ & 2.462 & 0.062 \\
\hline
\end{tabular}

